interosseous nerve ( 3 cases, twice bilaterally), and some very unusual ones such as the lateral antebrachial cutaneous nerve (1 case) and the sensory fibers of median nerve (1 case). NA also involved accessory spinal (2 cases, once bilaterally) and phrenic nerves ( 1 case bilaterally), both originating from cervical plexus. The EDX pattern of these nerve lesions consisted of unique or multiple extensive asymmetric inflammatory mononeuropathies with severe axonal loss and numerous denervation signs damage involving mostly the supra-scapular. On scapular MRI (available for $5 / 6$ patients), amyotrophy in at least one muscle was observed in all patients. Out of 26 nerves involved, after 12 months all had well recovered (above $3 / 5 \mathrm{MRC}$ scale).

Conclusion: HEV should be systematically screened when NA is suspected, whatever the severity, if the onset is less than 3 or 4 months (before IgMs antiHEV disappear). HEV-related NA appears to be frequently associated with a severe pattern, without modifying the recovery usually observed.

Disclosure of Interests: Romain Garofoli: None declared, Paul Seror: None declared, Jennifer Zauderer: None declared, Christelle Nguyen: None declared, François Rannou Grant/research support from: Pierre Fabre, Fidia, MSD, Pfizer, Bone Therapeutics, Expanscience, Grunenthal, Thuasne, Genévrier, Fondation Arthritis, Consultant of: Pierre Fabre, Fidia, MSD, Pfizer, Bone Therapeutics, Expanscience, Grunenthal, Thuasne, Genévrier, Speakers bureau: Pierre Fabre, Fidia, MSD, Pfizer, Bone Therapeutics, Expanscience, Grunenthal, Thuasne, Jean-Luc Drapé: None declared, Alexandra Roren: None declared, Marie-Martine Lefevre Colau: None declared

DOI: 10.1136/annrheumdis-2020-eular.2219

\section{AB0942 INFECTIOUS SPONDYLODISCITIS: TUBERCULOSIS VERSUS BRUCELLOSIS}

R. Grassa ${ }^{1}$, J. Anoun ${ }^{1}$, M. Thabet ${ }^{1}$, D. E. H. Abid ${ }^{1,2}$, F. Ben Fredj ${ }^{1,1}$, A. Rezgui ${ }^{1,2}$ C. Kechrid ${ }^{2} .^{1}$ Sahloul Hospital Tunisia, Internal Medicine, Sousse, Tunisia;

${ }^{2}$ Sahloul Hospital Tunisia, Sousse, Tunisia

Background: Infectious spondylodiscitis is a serious impairment that can compromise the functional and vital prognosis. The determination of the germ responsible is the key of the treatment.

Objectives: The objective of our work is to describe the epidemiological, clinical and evolutionary profile according to the germ responsible by comparing tuberculous and brucellar spondylodiscitis.

Methods: This is a retrospective study including 32 cases of spondylodiscitis with specific germs (Mycobacterium tuberculosis and Brucella) collected in an internal medicine department over a period of 18 years (2000-2018).

Results: These were 20 men and 12 women with an M / F ratio of 1.66. The average age of our patients was 50.63 [16-84]. The germ implicated was Koch's Bacillus in 11 patients $(34.38 \%$ ) and Brucella in 21 patients $(65.63 \%)$. The mean age for tuberculosis (TB) was 45.18 years versus 53.48 years for brucellosis. Spinal pain was the major symptom in the 02 groups. The deterioration in general condition was present in $80.95 \%$ for the brucellosis group versus $81.82 \%$ for the tuberculosis group.

Biological inflammatory syndrome was observed in $94.24 \%$ of the brucellosis group and $63.63 \%$ of the TBC group. The lumbar location was the most frequent in the 02 groups $(71.88 \%)$.It was a multifocal localization in $27.27 \%$ (TB) and $61.90 \%$ (Brucellosis) respectively. The imaging allowed the detection of para abscesses -vertebral in $54.55 \%$ for the TB group versus $23.81 \%$ for the brucellar group. An epiduritis was objectified in $36.36 \%$ of the TB group against $33.33 \%$ for that of brucellar. CT-guided biopsy was performed in $54.55 \%$ of tuberculosis patients compared to $47.62 \%$ in the other group. It was only positive in one case of brucellosis, whereas it allowed diagnosis in $36.36 \%$ of cases of TB.

The evolution after initiation of adequate antibiotic treatment was interspersed with neurological complications in the tuberculosis group in $18.18 \%$ of cases against $14.29 \%$ in the brucellosis group. Draining abscess was necessary in the tuberculosis group in $18.18 \%$ and in $9.52 \%$ of the brucellosis cases.

Conclusion: Our results show a higher frequency of neurological complications in tuberculosis forms. Vertebral biopsy is of no interest in Brucellar spondylodiscitis unlike tuberculosis forms where it allows the diagnosis.

References:

[1] Bousson,et al (2014). Infection rachidienne: du germe lent au staphylocoque doré. Revue Du Rhumatisme Monographies, 81(1), 27-35.

[2] Bierry, G., \& Dietemann, J.-L. (2012). Imagerie des spondylodiscites infectieuses. EMC - Radiologie et Imagerie Médicale - Musculosquelettique Neurologique - Maxillofaciale, 7(4), 1-16.

Disclosure of Interests: None declared

DOI: 10.1136/annrheumdis-2020-eular.4872

\section{AB0943 INFECTIOUS SPONDYLODISCITIS OF THE ELDERLY: CHARACTERISTICS AND OUTCOMES}

J. Mahbouba ${ }^{1}$, O. Jomaa ${ }^{1}$, S. Zrour ${ }^{1}$, I. Bejia ${ }^{1}$, M. Touzi ${ }^{1}$, N. Bergaoui ${ }^{1}$ on behalf of Rheumatology department. ${ }^{1}$ University Hospital of Monastir, TUNISIA, Rheumatology Department, Monastir, Tunisia

Background: Spondylodiscitis still frequent. It affects both old and young patients. It makes diagnostic and therapeutic difficulties.

Objectives: to identify the characteristics and outcomes of infectious spondylodiscitis (ISD) in patients over 65 years old.

Methods: A monocentric retrospective study including patients hospitalized for ISD in the rheumatology department of university hospital of Monastir,TUNISIA between January 2009 and August 2019.

Results: Among 70 patients with ISD, 21 (11 male, 10 female) or 30\% are over 65 years old. The average age was 70.6 years (65-82 years). History of diabetes $(n=9)$, hypertension $(n=9)$, hemodialysis $(n=5)$, heart disease $(n=5)$ were the most risk factors reported, while in younger patients, spinal surgery, epidural infiltrations and long-term general corticosteroid therapy were the main risk factors. The mean time for consultation was $142.3 \pm 73$ days longer than for younger patients. Fever was present in $0,14 \%$ of cases. Assessment time found that already 5 patients had paraplegia or spinal cord compression. 19/21 patients had epiduritis on spinal cord MRI. Soft tissue abscesses were present in close rate in both younger and old patients. Biological assessment showed an inflammatory syndrome and hyperleukocytosis in $92 \%$ and $38 \%$ of patients respectively (compared to $73 \%$ and $27 \%$ in younger patients). Germs ware identified in 14 patients $(47.3 \%)$. Common germs were the most involved (12 patients) while in younger patients, specific- germs were the most reported. Follow up has shown that neurological sequelae are more prevalent in elderly.

Conclusion: ISD in patients over 65 years old require a careful attension in therapeutic management given that age according to this study seems to influence the prognosis. In fact, these patients are more susceptible to disability due to neurological complications.

References: doi: 10.1016/j.medcli.2017.07.027.

doi: 10.1016/j.jinf.2008.02.005.

Acknowledgments: Rheumatology department

Disclosure of Interests: None declared

DOI: 10.1136/annrheumdis-2020-eular.6119

\section{AB0944 \\ TUBERCULOUS SEPTIC ARTHRITIS: CLINICAL FEATURES OF TWELVE CASES}

D. Khalifa ${ }^{1}$, K. Baccouche ${ }^{1}$, N. El Amri' ${ }^{1}$ H. Zeglaoui ${ }^{1}$, E. Bouajina ${ }^{1} .{ }^{1}$ Farhat Hached Hospital, Rheumatology Department, Susah, Tunisia

Background: Extrapulmonary forms of tuberculous septic arthritis account only for $1 \%$ of tuberculous infections. Although TB infection is rare in western countries, arthropathy is still a common problem in developing countries.

Objectives: Describe clinical features of tuberculous septic arthritis seen by the rheumatologist.

Methods: Retrospective descriptive study, lead in the rheumatology department of Farhat Hached Hospital, including medical files between 1999 and 2020. Data of patients diagnosed with tuberculous arthropathy were analysed.

Results: Twelve patients were diagnosed with tuberculous septic arthritis. Six men and women were enrolled with a sex ratio of 1 . The mean age of diagnosis was $47.5 \pm 16.16$ years. Mean delay of diagnosis was $12.83 \pm 15.12$ months. A triggering factor like a trauma was described in $8.3 \%$ and comorbidities were associated in $16.7 \%$ of the cases, mainly diabetes and chronic renal dysfonction. Type of pain was inflammatory in $91.7 \%$ of the time. The disease presented as a monoarthritis in $91.7 \%$ and an oligoarthritis in $8.3 \%$ of the cases. Chronic forms were observed in $91.7 \%$ and acute forms in $8.3 \%$ of the cases. Transmission was hematological in $60 \%$, directly inoculated in $20 \%$ and secondarily disseminated from another site in $20 \%$ of the cases. Arthritis affected the knees in $50 \%$, followed by the hips in $33.3 \%$ and then the ankles and wrist in $8.3 \%$ of the cases each. Fever was noted in $41.7 \%$ and general condition was altered in $50 \%$ of the patients. Chest radiographs showed the presence of infiltrates or micronodules in $33.3 \%$ of the patients. Bone erosions were detected in $66.7 \%$ of plain radiographs, while narrowing of the joint was seen in $83.3 \%$ and and juxta articular osteoporosis in $50 \%$ of the cases. MRI showed the presence of abces in $33 \%$ of the cases. The Mantoux test was positive in 2 cases, of which, one didn't receive the recommended neonatal vaccine. Culture was positive in the synovial fluid in $25 \%$, in sputum in $16.7 \%$, and in urine in $8.3 \%$ of the cases. Synovial biopsy was performed for all patients. It showed a non specific synovial inflammation in $50 \%$, granulomatous inflammation in $33.3 \%$ and caseous necrosis in $16.7 \%$ of the cases. Common quadri therapy was prescribed for all patients with a mean treatment duration of $11.41 \pm 1.37$ months. Surgery was performed in only two 
cases with an uncontrolled infection under antibiotics. Disseminated tuberculosis accured in $33.3 \%$, recurrence of the infection in the same site in $16.7 \%$ and extension to another articular localisation in $25 \%$ of the cases. One patient had a tuberculous meningoencephalitis leading to his death.

Conclusion: Tuberculous septic arthritis is difficult to diagnose and should be recalled especially in endemic countries when dealing with chronic monoarthritis. Synovial biopsy is needed most of the time to confirm the diagnosis. Treatment is long and the disease may be complicated with fatal disseminated forms.

References:

[1] Foocharoen C, Nanagara R, Foocharoen T, Mootsikapun P, Suwannaroj S, Mahakkanukrauh A. Clinical features of tuberculous septic arthritis in Khon Kaen, Thailand: a 10-year retrospective study. Southeast Asian J Trop Med Public Health. nov 2010;41(6):1438-46

Disclosure of Interests: None declared

DOI: 10.1136/annrheumdis-2020-eular.5664

\begin{tabular}{|l|l}
\hline AB0945 & CONTRIBUTION OF MICROBIOLOGICAL AND \\
ANATOMOPATHOLOGICAL EXAMINATIONS IN \\
THE DIAGNOSIS OF SPONTANEOUS PYOGENIC \\
SPONDYLODISCITIS IN ADULTS
\end{tabular}

I. Mahmoud ${ }^{1}$, M. Moalla ${ }^{1}$, A. Ben Tekaya ${ }^{1}$, S. Bouden ${ }^{1}$, R. Tekaya ${ }^{1}$, O. Saidane ${ }^{1}$, L. Abdelmoula ${ }^{1} .{ }^{1}$ Hospital Charles Nicolle, Rheumatology, TUNIS, Tunisia

Background: Pyogenic spondylodiscitis (SPD) is a serious infection of an intervertebral disc and/or adjacent vertebrae, that remains a topical problem in rheumatological practice. Early diagnosis and treatment are the only guarantees of a favorable outcome. Clinicians must strive to isolate the responsible bacteria in order to adapt the treatment, and thus reduce the risk of resistance and complications due to SPD itself, but also to the multiplication of probabilistic treatments.

Objectives: Our aim was to study the contribution of the different microbiological and anatomopathological examinations in the diagnosis of pyogenic SPD.

Methods: It was a descriptive study in a single rheumatology department. Data were collected retrospectively from observations of patients hospitalized in the past 20 years who have been diagnosed with pyogenic SPD. We excluded cases of tuberculous and brucellar SPD from our study because of their completely different histological and microbiological profiles.

Results: Twenty-two cases of pyogenic SPD were collected (14M/ 8F). The mean age of the population was 55.9 years $[29,80]$. A bacteriological survey including at least one cytobacteriological examination of the urine (CBEU), chest $\mathrm{X}$-rays and blood cultures allowed the identification of the bacteria in 16 cases (73\%). The most common site were bacteria was identified was blood culture in 7 cases, skin sample and urine collection in 2 cases each. Disco-vertebral puncture and biopsy (DVPB) was performed in 19 patients when there was no bacteria identification and/or when diagnosis of infectious SPD persisted doubtful. On histopathological examination, were described: an infiltrate and/or inflammatory changes without specificity signs in 7 patients and an appearance of chronic pyogenic SPD very likely in 12 patients. Bacteriological study of DVPB fluid or paravertebral abscesses sample helped to isolate bacteria in 4 patients. DVPB or abscesses puncture were contributing by histological and/or bacteriological examination in 12 patients $(63 \%)$

Infecting bacteria was identified in 14 patients (64\%). Gram-negative bacilli (GNB) and staphylococcus aureus were the most frequent germs ( 7 cases each) including 2 cases of co-infection. GNBs were represented by: Escherichia Coli and Enterobacter Cloacae in 2 cases each, Proteus Mirabilis, Serratia Marcescens and Klebsiella oxytoca in 1 case each. Clostridium clostridioforme and Lactococcus cremoris were isolated in 1 case each. For patients whose etiological investigation remained negative, SPD diagnosis was retained based on imaging (MRI) guided by anamnestic, clinico-biological and histopathological arguments.

Conclusion: SPD is a rare condition that needs to be treated rapidly. Once the diagnosis is suspected, bacteria must be isolated before starting any antibiotic therapy. Simple and non-invasive exams as blood cultures, CBUE and chest rays, should be undertaken first. In fact, these simple exams allowed a germ identification in $73 \%$ cases in our study. If doubt persist, DVPB could be contributive to the diagnosis.

References: None

Disclosure of Interests: None declared

DOI: 10.1136/annrheumdis-2020-eular.6272

\begin{tabular}{l|l}
\hline AB0946 & PARTICULARITY OF MULTIFOCAL INFECTIOUS \\
SPONDYLODISCITIS
\end{tabular}

S. Miladi ${ }^{1}$, K. Saadaoui ${ }^{1}$, A. Fazaa ${ }^{1}$, S. Mariem ${ }^{1}$, K. Ouenniche ${ }^{1}$, L. Souabni ${ }^{1}$, S. Kassab ${ }^{1}$, S. Chkili ${ }^{1}$, L. Zakraoui ${ }^{1}$, A. Laatar ${ }^{1} .{ }^{1}$ Mongi Slim Hospital, Rheumatology, Marsa, Tunisia
Background: Infectious spondylodiscitis is an infection of the intervertebral disc with damage to the vertebrae. Multiple stage involvement may lead to serious complications. Better understand of this infection is mandatory for optimal management.

Objectives: The objective of our study was to describe the clinical, biological aspects and the course of this disease.

Methods: This is a retrospective study including cases of infectious spondylo discitis collected in a single rheumatology center between 2002 and 2018. Multi focal spondylodiscitis was defined as an attainment of 2 or more disc levels. Clinical, biological and evolution of this disease were reported.

Results: Among the 52 cases of infectious spondylodiscitis collected, thirteen patients had multi-stage involvement of the spine. The sex ratio $M / F$ in this group $=0.44$ with an average age of $52 \pm 14.32$ years [ $31-76$ years]. Diabetes mellitus was observed in 3 patients, chronic renal failure in one case and immunosuppressive therapy in one case. At the moment of the diagnosis, inflammatory back pain was present in all patients, fever was noted in 8 patients $(61.5 \%)$ neurological deficit in 2 patients (15.4\%) and weight loss in 4 cases $(30.8 \%)$. The diagnosis time delay was $22 \pm 15.9$ weeks [ $2-60$ weeks]. The mean CRP was $70.16 \mathrm{mg} / \mathrm{L} \pm 55.5[3.4-178]$ and the $\mathrm{ESR}$ was on average $79 \mathrm{~mm} \pm 31.28[6$ 125]. Diagnosis was confirmed by MRI in all cases. A prevertebral abscess was observed in 7 cases (\%), an epiduritis in 5 patients (38.5\%) and a psoas abscess in two cases (15.2\%). The cervical spine was affected in 3 cases, the dorsa spine as well as the lumbar spine were affected in 12 cases. The involvment of 2 vertebral sites was the most frequent with 7 cases. A maximum of 5 site was noted in 3 cases. The thoracolumbar spine was the most affected with 11 cases $(84.6 \%)$, the cervico-thoraco-lumbar involvement was found in only one case $(7.7 \%)$. The bacteriological diagnosis was done by the disco-vertebral biopsy in 6 cases $(46.2 \%)$, the brucellar serology in 3 cases $(26.1 \%)$ and a strong presumption of tuberculosis (with positive IDR or Quantiferon) in 4 cases (30.8\%). Mycobaterium tuberculosis was isolated in 8 patients $(61.5 \%)$, brucella melitensis in 3 patients $(23.1 \%)$ and staphylococcus aureus was found in 2 cases $(15.4 \%)$. The outcome was favorable in all patients with appropriate antibiotic therapy after an average follow-up of 10.9 months \pm 2.19 .

Conclusion: Multifocal infectious spondylodiscitis is a serious disorder which mainly affects immunocompromised patients and the specific germs are the most incriminated.

Disclosure of Interests: None declared

DOI: 10.1136/annrheumdis-2020-eular.6172

\section{Pain in rheumatic diseases, including fibromyalgia}

\begin{tabular}{|l|l}
\hline AB0947 & RECIPROCAL IMPACT OF FIBROMYALGIA ON \\
DISEASE CHARACTERISTICS AND PHYSICAL AND \\
PSYCOLOGICAL DOMAINS IN SJOGREN SYNDROME: \\
CROSS SECTIONAL OBSERVATIONAL STUDY.
\end{tabular}

A. Capacci ${ }^{1}$, P. Rubortone ${ }^{1}$, V. Varriano ${ }^{1}$, A. Paglionico ${ }^{1}$, S. Perniola ${ }^{1}$ M. R. Gigante ${ }^{1}$, B. Tolusso ${ }^{1}$, S. Alivernini ${ }^{2}$, E. Gremese ${ }^{2} .{ }^{1}$ Fondazione $^{2}$ Policlinico Universitario A. Gemelli IRCCS, Rome, Italy; ${ }^{2}$ Fondazione Policlinico Universitario A. Gemelli IRCCS - Università Cattolica del Sacro Cuore, Rome, Italy

Background: Sjogren Syndrome (SS) is an autoimmune exocrinopathy, resulting mainly in ocular and oral dryness, with approximately half of patients displaying symptoms from different organ systems, further adding to the heterogeneous clinical phenotype of the disease. Fatigue and pain are common systemic symptoms in patients with primary SS and fibromyalgia is a frequent condition associated with chronic diseases.

Objectives: The aim of the study was to evaluate the impact of concomitant fibromyalgia in patients with Sjogren Syndrome in terms of clinical features and disease activity.

Methods: 50 patients with Sjogren Syndrome were enrolled in the study (100\% female, age: $53.7 \pm 13.2$ years and disease duration: $8.7 \pm 5.3$ years) $25(50.0 \%$ ) with concomitant fibromyalgia (SS/Fibro-group) and $25(50.0 \%$ ) without (SS-group). 36 patients with primary fibromyalgia (Fibro-group) were included as control group. At study entry, demographic, educational, life-style and clinical parameters were recorded for each patient. SS was diagnosed according to the American College of Rheumatology (ACR) classification criteria (1) and fibromyalgia was diagnosed according to criteria for fibromyalgia defined by ACR (2). Moreover, each patient with fibromyalgia, with and without concomitant SS, was asked to fill a self-reported questionnaire to assess the impact of Fibromyalgia on multiple physical and psycological domains (Italian-FIQR).

Results: Stratifying the study cohorts based on the demographic and life-style characteristics, no significant differences were found comparing SS-group, Fibrogroup and SS/Fibro-group. However, considering the different organ involvement 